\title{
Microscopic Entropy of the Black Ring
}

\author{
Michelle Cyrier, ${ }^{1}$ Monica Guica, ${ }^{1}$ David Mateos, ${ }^{2}$ and Andrew Strominger ${ }^{1}$ \\ ${ }^{1}$ Jefferson Physical Laboratory, Harvard University, Cambridge Massachusetts 02138, USA \\ ${ }^{2}$ Perimeter Institute for Theoretical Physics, Waterloo, Ontario N2L 2Y5, Canada
}

(Received 19 December 2004; published 18 May 2005)

\begin{abstract}
A surprising new seven-parameter supersymmetric black ring solution of five-dimensional supergravity has recently been discovered. In this paper, $\mathrm{M}$ theory is used to give an exact microscopic accounting of its entropy.
\end{abstract}

DOI: 10.1103/PhysRevLett.94.191601

PACS numbers: 11.25.Yb, 04.70.Dy

A major success of string theory is the statisticalmechanical explanation of the Bekenstein-Hawking entropy of certain supersymmetric spherical black holes [1]. A crucial implicit assumption is that a black hole is uniquely determined by its conserved asymptotic charges, because the macroscopic entropy is reproduced microscopically by counting Bogomol'nyi-Prasad-Sommerfield (BPS) states subject to the only constraint that they carry the same charges as the black hole.

Recently, a supersymmetric black ring has been discovered [2]. This is an asymptotically flat black hole solution of five-dimensional supergravity whose event horizon has topology $S^{1} \times S^{2}$. A more general seven-parameter supersymmetric black ring solution was found in [3-5]. Previous studies of non-BPS or singular black rings include [6]. Concentric black ring solutions have been found in $[5,7]$.

In $\mathrm{M}$ theory the solutions of $[2,3]$ correspond to supertubes [8], namely, to configurations with M5-branes wrapped around four-cycles of a six-torus and the fifth brane dimension forming a circle, or ring, stabilized by angular momentum in five-dimensional space-time. There is also M2-brane charge density distributed around the ring. The brane wrappings around the different cycles are summarized by the array

$$
\begin{array}{ccccccccc}
q_{1} & \mathrm{M} 2: & 1 & 2 & \ldots & \ldots & \ldots & \ldots & \ldots \\
q_{2} & \mathrm{M} 2: & \cdots & \ldots & 3 & 4 & \ldots & \ldots & \ldots \\
q_{3} & \mathrm{M} 2: & \cdots & \cdots & \cdots & \cdots & 5 & 6 & \ldots \\
p^{1} & \mathrm{M} 5: & \cdots & \cdots & 3 & 4 & 5 & 6 & \psi \\
p^{2} & \mathrm{M} 5: & 1 & 2 & \cdots & \cdots & 5 & 6 & \psi \\
p^{3} & \mathrm{M} 5: & 1 & 2 & 3 & 4 & \cdots & \cdots & \psi
\end{array}
$$

where $q_{A}$ and $p^{A}, A=\{1,2,3\}$, are the numbers of M2- branes and M5-branes, respectively, wrapping each cycle, and $\psi$ is the five-dimensional angular coordinate around the ring. The solution of the five-dimensional effective supergravity theory is reproduced in the appendix of this paper. It is characterized by seven independent parameters, which we choose to be the set of six brane numbers listed above, together with the angular momentum $J_{\psi}$ around the ring. However, the solution only carries five independent conserved charges, namely, the three M2 charges $q_{A}$ and the two angular momenta $J_{\psi}$ and $J_{\phi}$, where $J_{\phi}$ is the angular momentum in the plane orthogonal to the ring. The M5-branes do not give rise to conserved charges because they wrap a contractible cycle in space-time. Although the mass of the black ring is a conserved charge, it is not independent because saturation of the BPS bound implies $M=\sum q_{A}$.

It follows that, in addition to providing an example of a black hole with nonspherical topology, the black ring violates the uniqueness assumption above by two continuous parameters. It is therefore important to understand whether its entropy can still be exactly accounted for microscopically. In this Letter, $\mathrm{M}$ theory is used to provide such an accounting. A somewhat related approach to black ring entropy can be found in [9].

The Bekenstein-Hawking entropy, $S=\mathcal{A}_{\text {horizon }} /(4 G)$, of the black ring solution is given in [3] in the form

$$
S_{\mathrm{BR}}=2 \pi \sqrt{q_{1} q_{2} q_{3}-k_{1} k_{2} k_{3}-J_{\phi}^{2}-D\left(J_{\psi}-J_{\phi}\right)},
$$

where $k_{1} \equiv q_{1}-p^{2} p^{3}$ (and similarly for permutations of 1,2 , and 3) and $D \equiv p^{1} p^{2} p^{3}$. Replacing $J_{\phi}$ by its expression (A5) in terms of $q_{A}, p^{A}$, we get

$$
S_{\mathrm{BR}}=2 \pi \sqrt{\frac{D^{2}}{4}-D J_{\psi}-\frac{1}{4}\left(\left(p^{1} q_{1}\right)^{2}+\left(p^{2} q_{2}\right)^{2}+\left(p^{3} q_{3}\right)^{2}\right)+\frac{1}{2} D\left(\frac{q_{1} q_{2}}{p^{3}}+\frac{q_{2} q_{3}}{p^{1}}+\frac{q_{1} q_{3}}{p^{2}}\right)},
$$

where we have written everything in terms of the seven independent integers $q_{A}, p^{A}, J_{\psi}$. We wish to compare this expression with the formula we get from the microscopic computation.

Our starting point is Ref. [10], where a derivation of the entropy of a four-dimensional black hole in a Calabi-Yau $\times S^{1}$ compactification of M theory was given [11]. The construction involves an M5-brane wrapped about $\sum_{A} p^{A} \Sigma_{A}$, where $\Sigma_{A}$ 
is an integral basis of four-cycles in Calabi-Yau space. The resulting string in five dimensions is a chiral $(0,4)$ conformal field theory (CFT), whose left-moving central charge is given to leading order by $c_{L}=6 D$, where

$$
D=D_{A B C} p^{A} p^{B} p^{C}
$$

and $D_{A B C}$ are the triple intersection numbers of the $\Sigma_{A}$. For the case of a six-torus, relevant to the solutions of $[2,3]$, $D_{A B C}$ is equal to $1 / 6$ if $(A B C)$ form a permutation of (123) and zero otherwise. Therefore we can write

$$
c_{L}=6 D=6 p^{1} p^{2} p^{3} .
$$

Membrane charge arises as the momentum zero modes $q_{A}$ of a Narain lattice of scalars in the CFT. The left-moving oscillator number, denoted $\hat{q}_{0}$ in [10], was shown to be related to the left-moving momentum $q_{0}$ by [12]

$$
\hat{q}_{0}=q_{0}+\frac{1}{12} D^{A B} q_{A} q_{B}+\frac{c_{L}}{24}
$$

where $D^{A B}$ is the inverse of the matrix $D_{A B}$, defined as

$$
D_{A B}=D_{A B C} p^{C}=\frac{1}{6}\left(\begin{array}{ccc}
0 & p^{3} & p^{2} \\
p^{3} & 0 & p^{1} \\
p^{2} & p^{1} & 0
\end{array}\right) .
$$

The second term in $\hat{q}_{0}$ comes from the momentum carried by the Narain scalar zero modes, while the last term is the usual oscillator zero-point contribution to the momentum. These two terms are of the same order for a generic black ring because requiring the solution to be free of pathologies (e.g., closed timelike curves) imposes the condition $q_{1} \geq$ $p^{2} p^{3}$ (and similarly for permutations of 1,2 , and 3) [2,3]. To respect this inequality, $\sqrt{q} / p$ should be kept fixed in the large-charge limit, in which case the two terms above are of order $q^{3 / 2}$.

The entropy of excited states with left oscillator number $\hat{q}_{0}$ is given by the Cardy formula

$$
S=2 \pi \sqrt{c_{L} \hat{q}_{0} / 6}
$$

where $\hat{q}_{0}$ is the left-moving momentum available to be distributed among the oscillators [13].

In [10], a further $S^{1}$ compactification from five to four dimensions was performed, and four-dimensional black holes were made by wrapping the string around this $S^{1}$. The resulting macroscopic entropy agreed with the microscopic result following from Eq. (7). In the present context we do not wish to compactify to four dimensions. However, the intrinsic geometry of the M5-brane is identical: It still consists of a holomorphic surface times a circle. In [10], the M5 circle is topologically stable, whereas in the present case it is dynamically stabilized by the angular momentum. Since the effective $1+1$ CFT is obtained by Kaluza-Klein reduction of the M5-brane theory on the holomorphic surface, the result is the same $(0,4)$ theory as above.

Of course, the two M5 circles differ in their extrinsic geometry, namely, in the way they are embedded in spacetime, and this is reflected in the supergravity solution generated by the branes. The M5 circle in [10] is noncontractible, and hence the number of M5-branes can be determined by computing their net conserved charges at infinity. In the case of interest here, the M5 circle is contractible, and hence the net M5 charges at infinity vanish. The crucial point is, however, that the number of M5-branes that constitute the ring can still be determined completely unambiguously from the appropriate flux through any two-sphere that links the ring $[2,3,14]$.

In summary, we are proposing that the microscopic description of the black ring of $[2,3]$ is that of a ring of the same Calabi-Yau-wrapped M5-brane $(0,4)$ CFT encountered in [10]. Note that this is exactly in the spirit of [1], in that the microscopic theory is simply that living on the world volume of the branes that constitute the black hole in question. We emphasize that our proposal does not rely in any sense on a near-ring limit. The number of M2branes and the angular momenta are computed at infinity, as usual for conserved charges, and the flux that determines the number of M5-branes is computed through a sphere that may be taken to be arbitrarily large, the only constraint on it being a topological one, namely, that it has linking number one with the black ring horizon.

A highly nontrivial test of our proposal is the fact that the microscopic entropy exactly reproduces the Bekenstein-Hawking entropy. The left-moving momentum $q_{0}$ should equal $J_{\psi}$ up to a sign, since this is the momentum around the ring, and both are integrally quantized. Taking the sign such that $q_{0}=-J_{\psi}$ we find

$$
\begin{aligned}
\hat{q}_{0}= & -J_{\psi}+\frac{D}{4}+\frac{1}{2}\left(\frac{q_{1} q_{2}}{p^{3}}+\frac{q_{2} q_{3}}{p^{1}}+\frac{q_{1} q_{3}}{p^{2}}\right) \\
& -\frac{1}{4 D}\left(\left(p^{1} q_{1}\right)^{2}+\left(p^{2} q_{2}\right)^{2}+\left(p^{3} q_{3}\right)^{2}\right) .
\end{aligned}
$$

Substituting this expression into the Cardy formula (7) for the entropy and comparing with the supergravity result (2) we find perfect agreement.

We conclude with an observation about the infiniteradius limit of the ring, in which it becomes [2,3] the black string of [15]. In order to keep the resulting M2 charge densities per unit length finite, one must keep $q_{A} / R$ fixed. One must also keep $p^{A}$ fixed, since the M5-branes are extended along the circle direction. Finally, the angular momentum $J_{\psi}$ conjugate to $\psi$ must become a finite linear momentum density conjugate to $R \psi$, so $J_{\psi} / R^{2}$ must be kept fixed too. Thus, in the limit $R \rightarrow \infty$ the entropy density per unit length, $S / R$, remains finite, but the zeropoint contribution to $\hat{q}_{0}$, of order $R^{0}$, becomes subleading 
as compared to the two other terms in (5), of order $R^{2}$. This is consistent with the fact that the zero-point contribution could be neglected in [10], as reducing the resulting 5D string to four dimensions one obtains a black hole of the type considered in that reference.

We thank H. Elvang, R. Emparan, D. Jafferis, J. Maldacena, D. Marolf, H. Reall, and H. Verlinde for valuable discussions. This work was supported in part by DOE grant no. DE-FG02-91ER40654.

Appendix. - The five-dimensional metric has the form

$$
\begin{aligned}
d s^{2}= & -f^{2}(d t+\omega)^{2}+f^{-1} \frac{R^{2}}{(x-y)^{2}} \\
& \times\left[\frac{d y^{2}}{y^{2}-1}+\left(y^{2}-1\right) d \psi^{2}+\frac{d x^{2}}{1-x^{2}}+\left(1-x^{2}\right) d \phi^{2}\right]
\end{aligned}
$$

where $f^{-1}=\left(H_{1} H_{2} H_{3}\right)^{1 / 3}$,

$$
\begin{aligned}
H_{1}= & 1+\left(\frac{4 G}{\pi}\right)^{2 / 3} \frac{q_{1}-p_{2} p_{3}}{2 R^{2}}(x-y) \\
& -\left(\frac{4 G}{\pi}\right)^{2 / 3} \frac{p_{2} p_{3}}{4 R^{2}}\left(x^{2}-y^{2}\right)
\end{aligned}
$$

and $H_{2}$ and $H_{3}$ are given by obvious permutations.

The coordinates have ranges $x \in[-1,1], y \in$ $[-\infty,-1], \phi, \psi \in[0,2 \pi]$ and asymptotic infinity lies at $x \rightarrow y \rightarrow-1$. Further, for the black ring solution we have $\omega=\omega_{\phi} d \phi+\omega_{\psi} d \psi$, where

$$
\begin{aligned}
\omega_{\phi}= & -\frac{G}{2 \pi R^{2}}\left(1-x^{2}\right) \\
& \times\left[p^{1} q_{1}+p^{2} q_{2}+p^{3} q_{3}-D(3+x+y)\right], \\
\omega_{\psi}= & \frac{1}{2}\left(\frac{4 G}{\pi}\right)^{1 / 3}(1+y)-\frac{G}{2 \pi R^{2}}\left(y^{2}-1\right)\left[p^{1} q_{1}+p^{2} q_{2}\right. \\
& \left.+p^{3} q_{3}-D(3+x+y)\right],
\end{aligned}
$$

with $D=p^{1} p^{2} p^{3}$. The solution possesses two angular momenta as measured at infinity. In terms of the brane numbers and the radius $R$ they are given by

$$
\begin{gathered}
J_{\phi}=\frac{1}{2}\left(p^{1} q_{1}+p^{2} q_{2}+p^{3} q_{3}-D\right), \\
J_{\psi}=\left(\frac{\pi}{4 G}\right)^{2 / 3} R^{2}\left(p^{1}+p^{2}+p^{3}\right)+\frac{1}{2}\left(p^{1} q_{1}+p^{2} q_{2}\right. \\
\left.+p^{3} q_{3}-D\right) .
\end{gathered}
$$

Note that in terms of the charges used in [3] we have

$$
q_{i}=\left(\frac{\pi}{4 G}\right)^{2 / 3} Q_{i}^{E E M R}, \quad p^{i}=\left(\frac{\pi}{4 G}\right)^{1 / 3} q_{i}^{E E M R}
$$

[1] A. Strominger and C. Vafa, Phys. Lett. B 379, 99 (1996).

[2] H. Elvang, R. Emparan, D. Mateos, and H. Reall, Phys. Rev. Lett. 93, 211302 (2004).

[3] H. Elvang, R. Emparan, D. Mateos, and H. Reall, Phys. Rev. D 71, 024033 (2005).

[4] I. Bena and N.P. Warner, hep-th/0408106.

[5] J.P. Gauntlett and J.B. Gutowski, Phys. Rev. D 71, 045002 (2005).

[6] R. Emparan and H. S. Reall, Phys. Rev. Lett. 88, 101101 (2002); H. Elvang, Phys. Rev. D 68, 124016 (2003); H. Elvang and R. Emparan, J. High Energy Phys. 11 (2003) 035; R. Emparan, J. High Energy Phys. 03 (2004) 064.

[7] J.P. Gauntlett and J. B. Gutowski, Phys. Rev. D 71, 025013 (2005).

[8] D. Mateos and P. K. Townsend, Phys. Rev. Lett. 87, 011602 (2001); I. Bena and P. Kraus, Phys. Rev. D 70, 046003 (2004).

[9] I. Bena and P. Kraus, J. High Energy Phys. 12 (2004) 070.

[10] J. Maldecena, A. Strominger, and E. Witten, J. High Energy Phys. 12 (1997) 002.

[11] Although proper $S U(3)$ holonomy was assumed in [10], in the limit of large charges assumed here the corrections for a six-torus are subleading with respect to the terms below.

[12] The last zero-point term was omitted in [10] because it was subleading in the parameter range considered therein. We will come back to this below.

[13] We will assume that Cardy's formula can be applied despite the fact that $\hat{q}_{0} \gtrsim c_{L}$.

[14] R. Emparan, D. Mateos, and P. K. Townsend, J. High Energy Phys. 07 (2001) 011.

[15] I. Bena, Phys. Rev. D 70, 105018 (2004). 